\title{
Strong Modification of the Nonlinear Optical Response of Metallic Subwavelength Hole Arrays
}

\author{
J. A. H. van Nieuwstadt, ${ }^{1}$ M. Sandtke, ${ }^{1}$ R. H. Harmsen, ${ }^{1}$ F. B. Segerink, ${ }^{2}$ J. C. Prangsma, ${ }^{1}$ S. Enoch, ${ }^{3}$ and L. Kuipers ${ }^{1, *}$ \\ ${ }^{1}$ FOM Institute for Atomic and Molecular Physics (AMOLF), Kruislaan 407, 1098 SJ, Amsterdam, the Netherlands \\ ${ }^{2}$ Applied Optics Group, MESA ${ }^{+}$Research Institute and Department of Science and Technology, University of Twente, \\ P.O. Box 217, 7500 AE, Enschede, the Netherlands \\ ${ }^{3}$ Institut Fresnel, CNRS 6133, Campus de St. Jérôme, Case 161, 13397 Marseille Cedex 20, France
}

(Received 9 June 2006; published 6 October 2006)

\begin{abstract}
The influence of hole shape on the nonlinear optical properties of metallic subwavelength hole arrays is investigated. It is found that the amount of second harmonics generated can be enhanced by changing the hole shape. In part this increase is a direct result of the effect of hole shape on the linear transmission properties. Remarkably, in addition to enhancements that follow directly from the linear properties of the array, we find a hot hole shape. For rectangular holes the effective nonlinear response is enhanced by more than 1 order of magnitude for one particular aspect ratio. This enhancement can be attributed to slow propagation of the fundamental wavelength through the holes which occurs close to the hole cutoff.
\end{abstract}

DOI: $10.1103 /$ PhysRevLett.97.146102

Metal nanostructures are known to greatly enhance electromagnetic fields in certain geometries. Therefore they can also boost nonlinear optics. Surface-enhanced Raman scattering (SERS) [1], for instance, makes use of nobel metal nanoparticles to amplify the spectroscopic Raman signature of even a single molecule [2]. Sharp nanosize tips [3] provide a more controlled route to enhance the optical field. Another powerful example of nonlinear enhancement is a single circular hole, surrounded by an ordered plasmonic structure [4]. In addition to the use of single structures, ensembles of multiple nanostructures or extended metal objects can also be used to induce nonlinear enhancement randomly positioned metal nanoclusters show nonlinear effects [5]. A popular example of multiple nanostructures acting together is a subwavelength hole array which exhibits extraordinary optical transmission [6] in the linear regime. Calculations indicate that the local field enhancement associated with the transmission through the holes is exceptionally large [7]. Blair and co-workers showed that second-harmonics generation (SHG) on a hole array is possible [8].

The shape of a metal nanostructure influences its field enhancement, and thus potentially its nonlinear behavior. Shape may explain why 1 out of 100 to 1000 nanoparticles exhibits high SERS enhancement efficiencies and are called "hot particles" [2]. Shape can also break mirror symmetry to allow SHG [8,9]. Changing the shape of subwavelength holes in periodic arrangements affects the linear transmission properties of the array [10-13]. An enhancement of the linear transmission through changes in hole shape would lead to higher intensities in the holes and as a consequence to a predictable increase in, for example, SHG. Recent work has described SHG from a nonlinear medium combined with a coaxial hole array [14]. No studies have been performed on the effects of shape on the actual nonlinear response of subwavelength hole arrays
PACS numbers: 81.07. $-\mathrm{b}, 42.79 . \mathrm{Dj}, 71.36 .+\mathrm{c}, 78.66 . \mathrm{Bz}$

itself, or in other words on their effective nonlinear susceptibility.

In this Letter we present a study of the nonlinear response of a metallic subwavelength air hole array by measuring SHG. As the aspect ratio (AR) of rectangular subwavelength holes is varied, an increased transmission of the fundamental light and a concomitant increase of local fields lead to an expected increase in SHG. From the combination of the SHG signal and the linear transmission properties of the array, we have determined the effective nonlinear susceptibility $\left(\chi_{\text {eff }}^{(2)}\right)$ of the arrays as a function of AR. Surprisingly, we find that a "hot" hole shape exists for which the $\chi_{\mathrm{eff}}^{(2)}$ is enhanced by more than 1 order of magnitude. We are able to attribute this enhancement to the cutoff condition of the rectangular holes, acting as waveguides for the fundamental wavelength used. The cutoff condition leads to slow light propagation through the holes, resulting in an enhancement of the nonlinear effects.

Multiple subwavelength hole arrays are studied, each consisting of $20 \times 20$ rectangular holes in a square lattice [Fig. 1(a)]. The holes are milled in an optically thick Au

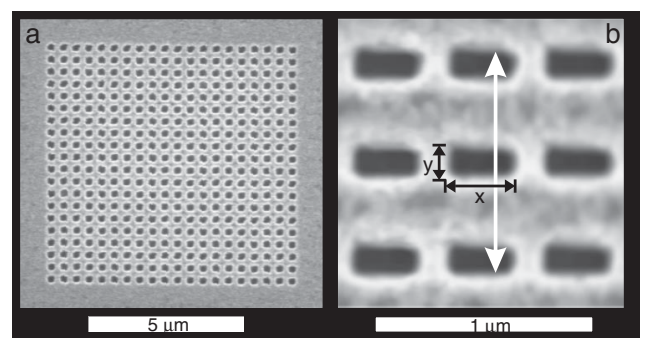

FIG. 1. (a) Scanning electron microscope (SEM) micrograph of an array of square holes. (b) SEM micrograph of an array with $\mathrm{AR}=x / y=2.0$, where $x$ and $y$ (black arrows) are defined as the hole dimensions perpendicular and parallel to the polarization of the incident light (white arrow), respectively. 
film (thickness $160 \mathrm{~nm}$ ) on a glass substrate. Both the hole area and lattice periodicity are kept fixed at $3.4 \times 10^{4} \mathrm{~nm}^{2}$ and $410 \mathrm{~nm}$, respectively. The different arrays contain holes with different dimensions, ranging from $180 \times$ $190 \mathrm{~nm}^{2}$ to $280 \times 120 \mathrm{~nm}^{2}$. As the extraordinary transmission through the array, and therefore its linear properties, follows a power dependence on hole area with a high power [15], care was taken to keep the hole area constant. The aspect ratio of the holes is defined as $\mathrm{AR}=x / y$, with $x$ and $y$ the hole dimensions perpendicular and parallel to the polarization of the incident fundamental light, respectively [Fig. 1(b)]. This way, an AR range of 0.42 to 2.4 is explored.

The sample is illuminated with bandwidth-limited light pulses from a Ti:sapphire laser (pulse duration $80 \mathrm{fs}$, repetition rate $80 \mathrm{MHz}$, and wavelength tunable between 750 and $830 \mathrm{~nm}$ ). This fundamental beam is focused onto the $\mathrm{Au}$ side of the sample with a numerical aperture (NA) of 0.07 . An achromatic lens $(\mathrm{NA}=0.3)$ on the glass side of the sample collects only light from the zeroth diffraction order, which is spectrally analyzed for the polarization directions along $x$ and $y$ (spectral resolution $0.3 \mathrm{~nm}$ ). To avoid damage of the detector by the transmitted fundamental light, we use a colored-glass filter with a transmission factor $T=0.40$ for the SHG light and $T=2.4 \times 10^{-9}$ for the fundamental light.

A typical spectrum is given in Fig. 2(a). The sharp peak at $\lambda=830 \mathrm{~nm}$ corresponds to the fundamental wavelength. The inset reveals a second peak at $415 \mathrm{~nm}$, accompanied by a broad background centered at $450 \mathrm{~nm}$. The shape of the latter is affected by the colored-glass filter and actually extends beyond $500 \mathrm{~nm}$. The background has a cubic dependence on the incident fundamental power [Fig. 2(b)] and is attributed to three-photon luminescence [16]. This power dependence is different than that obtained previously in different Au nanostructures [17], indicating a different physical origin. For the determination of the intensity of the small sharp peak, this background is always subtracted. To prove that the small sharp peak is due to SHG in the hole array, we perform three control experiments. First, we tune the fundamental wavelength and observe that the small peak is always exactly at half the fundamental wavelength. Second, the quadratic dependence on the fundamental input power is verified [Fig. 2(b)] [18]. Third, the SHG signal at $415 \mathrm{~nm}$ from both a plain glass substrate and a plain Au film is checked to stay below noise level. It may seem surprising that an SHG signal is obtained from this centrosymmetric structure using excitation and detection angles that are perpendicular to the sample $[8,19]$. We remark, however, that symmetry breaking at an interface can be sufficient to generate a scattered beam of second-harmonics $(\mathrm{SH})$ light [20], which can be detected if the collection NA is large enough to include such scattering angles [4].

By investigating arrays with different hole shapes, we find that the influence of the AR on the amount of SHG collected is very large (Fig. 3). Both the fundamental transmission and the SHG exhibit a general increase as the AR is increased from 0.42 to 2.4. Moreover, a distinct enhancement in SHG, centered at $\mathrm{AR}=2.0$ with a width of 0.2 in units of AR, of more than 1 order of magnitude is observed. The influence of AR on the linear transmission of the fundamental is consistent with previous work [21].

For a better understanding of the behavior of the SHG, we approximate the hole arrays as a slab of effective nonlinear medium that generates second harmonic; i.e., we ignore a probable intensity distribution in the $(x, y)$ plane of the metal array. The nonlinear properties of each array can be characterized by the second-order nonlinear susceptibility $\chi_{i j k}^{(2)}$ [18]. In the case of SHG, the index $i \in\{x, y, z\}$ corresponds to the vector components of the electric field of the $\mathrm{SH}$ wave, and $j=k$ corresponds to the vector components of the electric field of the fundamental wave. Thus, $\chi_{y y y}^{(2)}$ and $\chi_{x y y}^{(2)}$ determine the amplitudes of SH waves with $y$ and $x$ polarization, respectively, that are generated by a fundamental wave that is polarized in $y$ direction.

The interaction length of the electric field with the array is equal to the film thickness, which is small compared to the wavelength. Therefore, perfect phase matching can be assumed. Because of the low SHG conversion, the nondepleted pump approximation is justified. Under these assumptions, Maxwell's equations can be analytically a

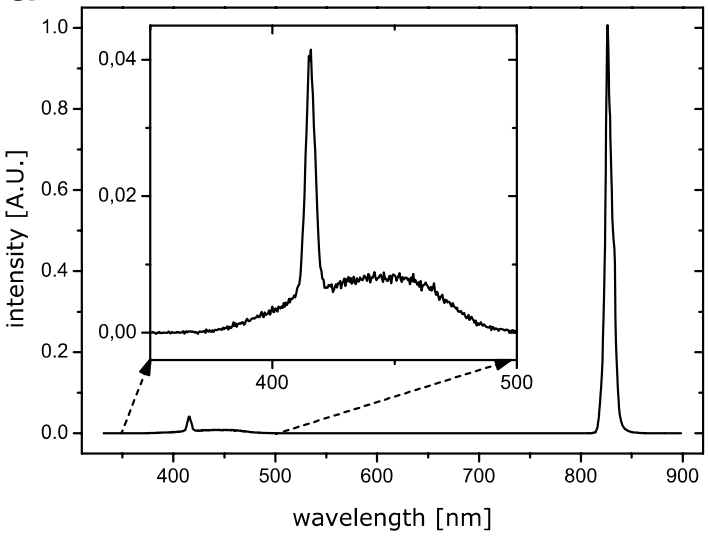

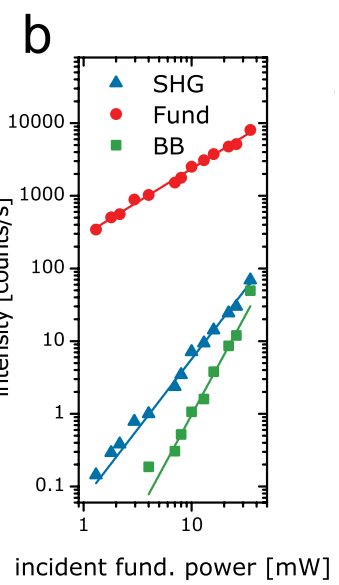

FIG. 2 (color). (a) Transmission of the fundamental light at $830 \mathrm{~nm}$, strongly attenuated by colored-glass filters. The small sharp peak at $415 \mathrm{~nm}$ visible in the inset is secondharmonics light. The broad background (BB) that is also visible in the inset is attributed to three-photon luminescence. (b) Double-log plot of the SHG power (triangles), transmitted fundamental power (circles), and the broad background (squares) as a function of the incident fundamental input power. The solid lines are power-law fits. The fitted exponents are $1.9 \pm 0.1,0.9 \pm$ 0.1 , and $2.7 \pm 0.2$ for the SHG, the fundamental, and the background, respectively. 


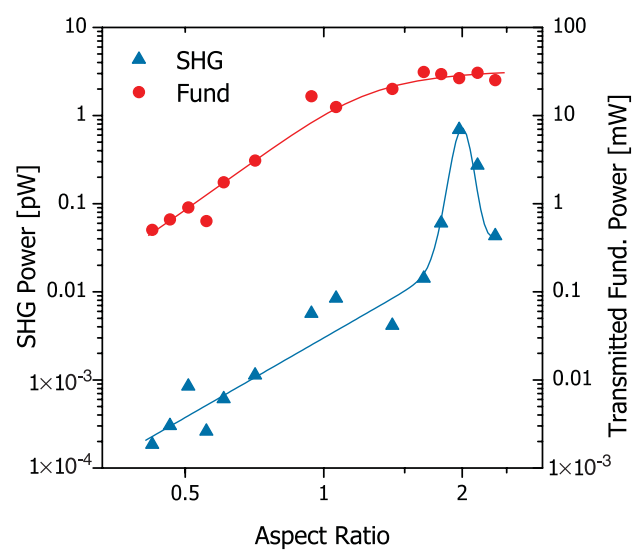

FIG. 3 (color). Double-log plot of the SHG power (triangles) and fundamental light transmission (circles) as a function of hole AR for equal polarizations. The SHG increases by 2 orders of magnitude and has a peak of almost another 2 orders of magnitude at $\mathrm{AR}=2.0$. The fundamental transmission also increases with the AR. The solid lines are guides to the eye.

solved to calculate the SHG yield of a medium in which all the beams can exhibit attenuation [18]. Consequently, if the SHG yield is known, the solution can be used to calculate the effective $\chi_{i j k}^{(2)}$ of the crystal:

$$
\left[\chi_{i j k}^{(2)}\right]_{\mathrm{eff}}^{2}=\frac{\mathcal{P}_{2 \omega}}{\mathcal{P}_{\omega}^{2}} \frac{\epsilon_{0} \epsilon_{\omega} \sqrt{\epsilon_{2 \omega}} c \lambda_{\omega}^{2} O}{2 \pi^{2}}\left[\frac{\alpha_{\omega}-\frac{1}{2} \alpha_{2 \omega}}{e^{-\alpha_{\omega} d}-e^{-(1 / 2) \alpha_{2 \omega} d}}\right]^{2},
$$

where $\mathcal{P}_{\omega}$ is the incident fundamental power, and $\mathcal{P}_{2 \omega}$ the SHG power on the output side of the crystal. $O$ is the surface of the array, $\epsilon_{0}$ the permittivity of free space, $c$ the speed of light in vacuum, and $\lambda_{\omega}$ the fundamental wavelength. $\epsilon_{\omega}$ and $\epsilon_{2 \omega}$ are the linear dielectric constants of the medium at fundamental and SH wavelength, respectively. The effective dielectric constants for the arrays are unknown and not trivial to determine. To obtain these values, we treat the subwavelength holes as waveguides with the same lateral dimensions. For these waveguides we calculate the wave vectors of the lowest order modes (see below) from which the linear dielectric constants follow. Please note that our results are hardly affected by the particular choice of $\epsilon_{\omega}$ and $\epsilon_{2 \omega} . \alpha_{\omega}$ and $\alpha_{2 \omega}$ are the linear attenuation coefficients of the medium at fundamental and SH wavelength, respectively, which can be determined from the measured linear transmission coefficient of the array using $\alpha=-\ln (T) / d$. Here, $d$ and $T$ are the Au film thickness and the transmission coefficient, respectively. By determining the $\left|\chi^{(2)}\right|_{\text {eff }}$ in this way we ignore possible changes in the shape of the intensity distributions in the plane of the array. However, effects caused by an increase in local intensities resulting merely from an enhanced linear transmission due to hole shape drop out. As a result we obtain the effect of hole shape on the effective nonlinear response.
Elements of the nonlinear susceptibility are plotted as a function of AR in Fig. 4. $\left|\chi_{\text {yyy }}^{(2)}\right|_{\text {eff }}$ is determined from the data presented in Fig. 3, and $\left|\chi_{x y y}^{(2)}\right|_{\text {eff }}$ results from an additional data set that was acquired in the same way as Fig. 3 but with the analyzing polarizer set perpendicularly to the polarization of the incident fundamental light. We have normalized the effective nonlinear susceptibility to the $\left|\chi^{(2)}\right|_{\text {eff }}$ at $\mathrm{AR}=0.94$ in order to focus on the differences due to the variation in hole shape and to be insensitive to a nonunity collection efficiency that might affect the absolute value of $\left|\chi^{(2)}\right|_{\text {eff }}$. Between $\mathrm{AR}=0.4$ and $\mathrm{AR}=$ 1.5 no clear trend is discernible in the effective nonlinear susceptibility. This indicates that the gradual increase of the SHG signal over 2 orders of magnitude, shown in Fig. 3, is primarily explained by linear transmission effects only. However, we observe a large increase of the $\left|\chi^{(2)}\right|_{\text {eff }}$ around $\mathrm{AR}=2$, indicating a strong enhancement of the nonlinear response.

In order to understand the enhancement it is useful to consider the calculated wave vectors of the guided modes in the array, for the fundamental light for the different hole shapes. We performed calculations with a Fourier modal method in which all modes, including the evanescent modes, are obtained through an eigenvalue problem of the Fourier components of the fields [21,22]. Figure 5 shows the real and imaginary parts of the wave vector $k_{g}$ for the different AR investigated normalized to the wave vector of the fundamental beam in vacuum. It is clear that for the modes in the real metal there is no clear distinction between propagating $\left(k_{g}\right.$ real) and nonpropagating $\left(k_{g}\right.$ fully imaginary) modes, unlike the modes for a perfectly conducting metal, which, in the geometries considered here, are fully imaginary. This observation has previously been made for cylindrical waveguides [23] and rectangular

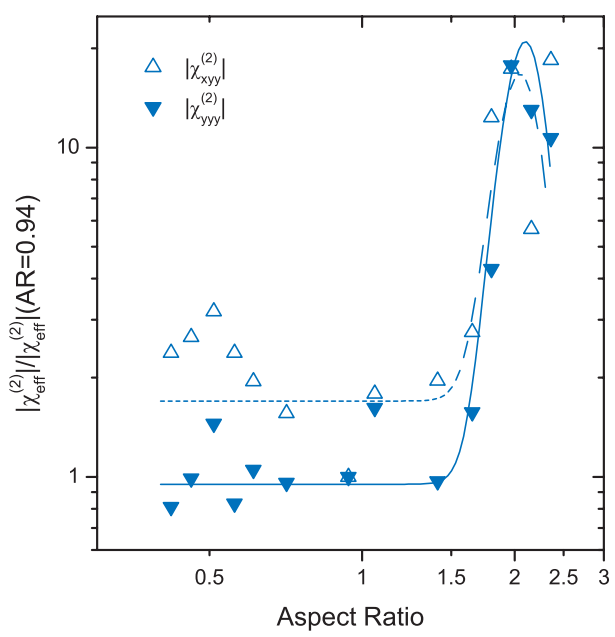

FIG. 4 (color). Double-log plot of the normalized effective nonlinear susceptibility $\left|\chi_{i j k}^{(2)}\right|$ as a function of the AR. $\left|\chi_{y y y}^{(2)}\right|$ (solid triangles) and $\left|\chi_{x y y}^{(2)}\right|$ (open triangles) correspond to SHG with $y$ and $x$ polarization, respectively, both with the fundamental polarized along $y$. The lines are guides to the eye. 


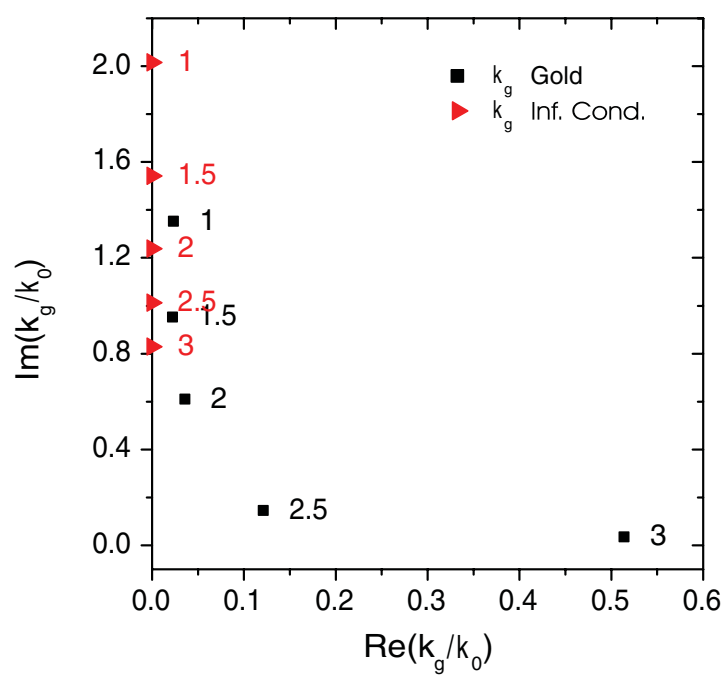

FIG. 5 (color). Calculated imaginary part vs the real part of the propagation constant perpendicular to the metal film of the fundamental modes for subwavelength rectangular holes integrated in a periodic array with dimensions of the experimentally investigated arrays, at an incident wavelength of $830 \mathrm{~nm}$. The AR of the holes is indicated by the numbers. The square and triangular markers correspond to gold and infinitely conducting structures, respectively.

holes [24]. We can distinguish two regimes. The first regime starts at $\mathrm{AR}=1$ and runs roughly until $\mathrm{AR}=2$; here the imaginary part of $k_{g}$ is dominant; i.e., the modes excited with a free space wavelength of $830 \mathrm{~nm}$ are mainly evanescent. On the other hand, the second regime, starting at $\mathrm{AR}=2.5$ and extending to higher $\mathrm{AR}$, shows modes with a dominance of the real part of $k_{g}$. Here, the modes are propagating. In between the evanescent and the propagating regime will be the cutoff region for a single hole. It has also been pointed out that single rectangular holes exhibit a resonance in their transmission close to cutoff with large associated field enhancements [25]. It is clear from Fig. 5 that the cutoff region is exactly the region where we observe the enhancement of the $\left|\chi^{(2)}\right|_{\text {eff }}$ by 1 order of magnitude. When a waveguide is close to cutoff, the modes in that guide will have a low group velocity (see, e.g., [23]). It has been shown theoretically that a reduction in group velocity leads to enhanced nonlinear responses due to an effective increase of light-matter interactions [26]. We suggest that these slow modes for the fundamental wavelength cause the sharp increase in the $\left|\chi^{(2)}\right|_{\text {eff }}$.

We checked our hypothesis by changing the wavelength of the fundamental and the periodicity of the arrays. The enhancement of $\left|\chi^{(2)}\right|_{\text {eff }}$ at AR $=2$ exhibits a peak with a FWHM of $80 \mathrm{~nm}$ around $830 \mathrm{~nm}$. We find that the strong increase in $\left|\chi^{(2)}\right|_{\text {eff }}$ around $\mathrm{AR}=2$ cannot be shifted, within the experimental error, by changing the lattice periodicity. This strongly suggest that the increase in $\left|\chi^{(2)}\right|_{\text {eff }}$ at $\mathrm{AR}=2$ is strongly linked to the incident fundamental and is a property of the single holes rather than of the array as a whole.
In conclusion, we have shown by means of SHG that the nonlinear optical properties of a metallic subwavelength hole array can be strongly modified by tailoring the shape of the holes. Increasing the hole AR results in an increase of the SHG yield, resulting from increasing local fields and an increase in linear transmission. Moreover, for holes with $\mathrm{AR}=2.0$, we observe an enhancement of 1 order of magnitude in the effective second-order susceptibility of the array. We attribute this enhancement to a cutoff behavior in the individual holes, leading to slow modes inside the holes. Our findings are promising for improving nonlinear applications such as SERS, all-optical switching, and ultrahigh resolution near-field microscopy.

This work was made possible by the facilities of the Amsterdam nanoCenter. The work is part of the research program of the Stichting voor Fundamenteel Onderzoek der Materie (FOM), which is financially supported by the Nederlandse organisatie voor Wetenschappelijk Onderzoek (NWO). Support of the EC-funded project PHOREMOST (No. FP6/2003/IST/2-511616) is gratefully acknowledged. The authors thank Andrei Petukhov for useful discussions.

*Corresponding author. Electronic address: 1.kuipers@ amolf.nl

[1] R. Chang and T. Furtak, Surface Enhanced Raman Scattering (Plenum, New York, 1981).

[2] S. Nie and S. R. Emory, Science 275, 1102 (1997).

[3] A. Bouhelier et al., Phys. Rev. Lett. 90, 013903 (2003).

[4] A. Nahata et al., Opt. Lett. 28, 423 (2003).

[5] S. I. Bozhevolnyi et al., Phys. Rev. Lett. 90, 197403 (2003).

[6] T. W. Ebbesen et al., Nature (London) 391, 667 (1998).

[7] L. Salomon et al., Phys. Rev. Lett. 86, 1110 (2001).

[8] M. Airola, Y. Liu, and S. Blair, J. Opt. A Pure Appl. Opt. 7, S118 (2005).

[9] B. Lamprecht et al., Appl. Phys. B 68, 419 (1999).

[10] K. J. Klein Koerkamp et al., Phys. Rev. Lett. 92, 183901 (2004).

[11] R. Gordon et al., Phys. Rev. Lett. 92, 037401 (2004).

[12] D. van Labecke et al., J. Microsc. 213, 140 (2004).

[13] W. Fan et al., Phys. Rev. Lett. 94, 033902 (2005).

[14] W. Fan et al., Nano Lett. 6, 1027 (2006).

[15] K. L. van der Molen et al., Appl. Phys. Lett. 85, 4316 (2004).

[16] C. K. Chen et al., Phys. Rev. Lett. 46, 145 (1981).

[17] M. Beversluis et al., Phys. Rev. B 68, 115433 (2003).

[18] R. L. Sutherland, Handbook of Nonlinear Optics (Marcel Dekker, New York, 1996).

[19] F. Brown et al., Phys. Rev. Lett. 14, 1029 (1965).

[20] S. Roke et al., Phys. Rev. B 70, 115106 (2004).

[21] K.L. van der Molen et al., Phys. Rev. B 72, 045421 (2005).

[22] L. F. Li, J. Opt. Soc. Am. A 14, 2758 (1997).

[23] L. Novotny et al., Phys. Rev. E 50, 4094 (1994).

[24] R. Gordon and A. G. Brolo, Opt. Express 13, 1933 (2005).

[25] F. Garcia-Vidal et al., Phys. Rev. Lett. 95, 103901 (2005).

[26] M. Soljačić et al., Nat. Mater. 3, 211 (2004). 